\title{
Measurement of Wear in Orthopedic Prosthesis
}

\author{
B. SAGBAS ${ }^{a, *}$ AND M. NumAn DURAKBASA ${ }^{b}$ \\ ${ }^{a}$ Department of Mechanical Engineering, Yildiz Technical University, Istanbul, Turkey \\ ${ }^{b}$ Department of Interchangeable Manufacturing and Industrial Metrology \\ Vienna University of Technology, Austria
}

\begin{abstract}
Wear in orthopedic prosthesis has been the main limiting factor of total joint replacement's service life. Wear analysis of total joint replacements is essential for determining failure mechanism, prediction of wear and longevity of implants. So it would be possible to improve design, material and manufacturing quality and service life of these devices. Radiographic, gravimetric, volumetric and optical techniques are current methods for measuring of wear in retrieved implants. Gravimetric method is the standardized method for quantifying wear volumes of total joint prosthesis. Although this method is effective for determining experimental wear volume in simulated conditions, it is not suitable for assessing the clinically retrieved prosthesis for which there is no pre-wear data available. Each of the wear measuring methods has advantages and limitations. Researches have been going on for developing effective methods. In this paper the techniques that are currently used for evaluating wear of orthopedic prosthesis like gravimetry, coordinate measuring machine, micro computed tomography, and digital photogrammetry are explained. The literature works and new trends in metrological assessment of wear are reviewed.
\end{abstract}

PACS: 06.90. $+\mathrm{v}$

\section{Introduction}

Total joint replacements have been applied for the patients that are affected by osteoarthritis [1]. Although these replacements improve life quality of the patients, their premature failure causes lots of problem for both patients and surgeons. For articulating surfaces wear is the primary failure factor that limits the service life of the prosthesis $[2,3]$. Articulating surface materials and the other components of artificial knee and hip joints can be seen in Figure 1.
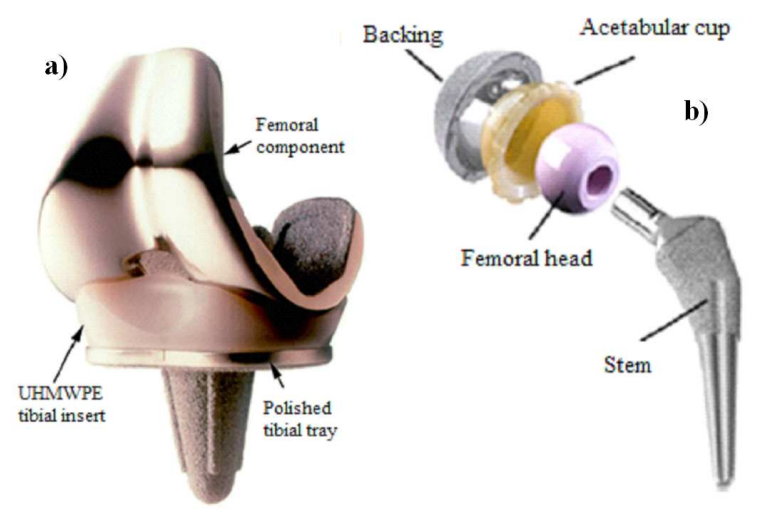

Fig. 1. Artificial joint components: (a) total knee joint, (b) total hip joint [7, 18].

The wear debris generated from bearing materials could cause aseptic loosening, osteolysis and at the end implant loss. Although the researches have been done for

\footnotetext{
* corresponding author; e-mail: bzeybek@yildiz.edu.tr
}

determining the wear behavior of the total joint replacements in order to improve design, material and manufacturing quality and service life of these joints, it is still unknown phenomena how to design wear resistant artificial joint parts and select ideal material pairs for these kinds of replacements. Therefore examining the wear characteristics of the prosthesis materials both for in vivo clinical applications and in vitro laboratory simulations is still one of the most important topics for researchers [4-7]. In Fig. 2, a retrieved UHMWPE tibial insert can be seen. The worn and delaminated areas on the surface of this component are apparent.

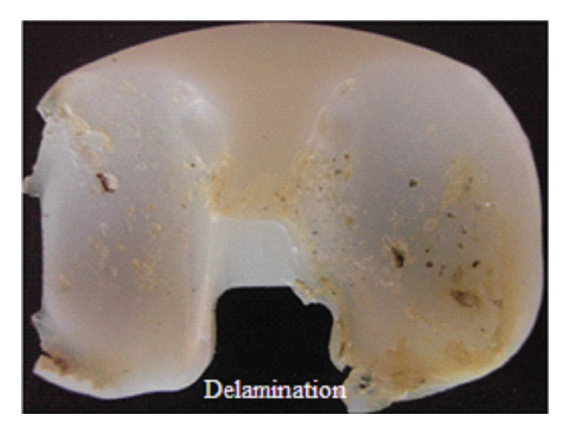

Fig. 2. Example of failure on retrieved ultra high molecular weight polyethylene (UHMWPE)total knee joint component.

Radiographic, gravimetric, volumetric and optical techniques are current methods for measuring and evaluating of wear in total joint replacement components. Gravimetric method is the standardized method for quantifying wear volumes of total joint prosthesis. Although this method is effective for determining exper- 
imental wear volume in simulated conditions, it is not suitable for assessing the clinically retrieved prosthesis for which there is no pre-wear data available [8-12]. Radiographic techniques allow us estimation about femoral head migration into the cup. These techniques can be applied before explantation surgery of the prosthesis. Because of the 2D nature of many radiographic methods it is sometimes difficult to position the implants correctly, so this may cause mischaracterization of the wear. Availability of initial radiographs, coarse image resolution, and assumptions about wear pattern are the limiting factors for these techniques $[8,13,14]$. Volumetric method has recently been an alternative method to gravimetric method [10]. Volumetric wear rates can be directly defined by using the coordinate measuring machines (CMMs) and micro computed tomography (micro-CT, $\mu$-CT) techniques $[8,10]$. The use of CMM is standardized by ISO 14242-2:2000. Besides quantification of wear volume, this method makes possible the assessment of wear scars [10]. Micro-CT is one of the high resolution measurement techniques. Both in CMM and micro-CT techniques the wear volume of retrieved prosthesis is defined by comparing the idealized unworn 3D geometry data with worn geometry data. Lots of papers were published for development of a reliable wear assessment method by using CMM and micro-CT techniques [10, 11, 13, 15-18]. An example for measurement of an UHMWPE hip joint component can be seen in Fig. 3 .

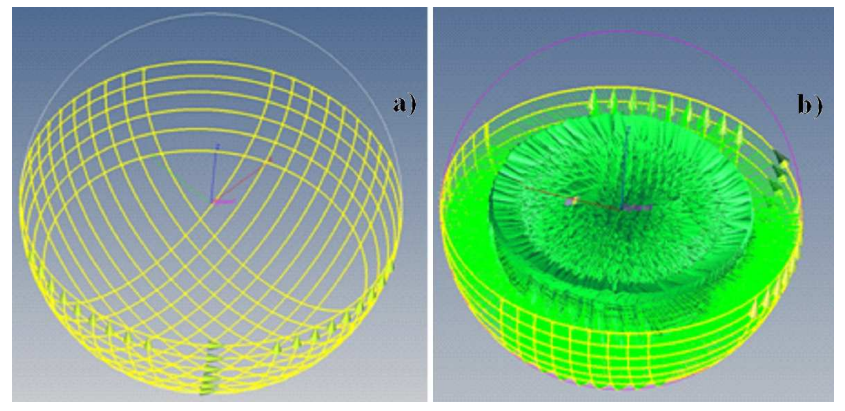

Fig. 3. CMM measurement of bearing surface on a UHMWPE hip joint component. (a) Defining measurement paths, (b) inner surface of the prosthesis saturated with measurement points.

Each of the wear measurement method has advantages and limitations. Researches have been going on for developing effective methods. In this paper the techniques that were currently used for evaluating wear of orthopedic prosthesis like gravimetry, CMM, $\mu$-CT, and digital photogrammetry are explained. The literature works and new trends in metrological assessment of wear are reviewed.

\section{Gravimetric method}

This is the most common and standardized method to evaluate wear volume in hip joints. In this method specimen weight is measured before and after wear test and the difference of the two measurement values are calculated. ISO 14242-2:2000 requires a balance with an accuracy of $\pm 0.1 \mathrm{mg}$. For reference purposes a loaded but not articulating control specimen is needed for determining fluid sorption of specimen. Gravimetric wear can be calculated as follows:

$$
W_{n}=W_{a n}+S_{n} .
$$

Here $W_{n}$ is the net mass loss after $n$ cycles of loading, $W_{a n}$ is the average uncorrected mass loss, $S_{n}$ is the average increase in mass of the control specimen over the same period.

By using the least squares linear fit relationship between $W_{n}$ and the number of loading cycles $n$ the average wear rate $a_{\mathrm{G}}$ can be calculated as follows:

$$
W_{n}=a_{\mathrm{G}} n+b .
$$

However accurate results it gives, this method can only be applied during in vitro testing of new hip prostheses [10]. It does not give any information about the worn surfaces and plastic deformation of the implant material. Because of not having initial weight measurement values, it is not possible to define wear of retrieved implants [12]. Besides this both for in vivo and in vitro applications, material transfer from the metal component or bone cement that are attached into the UHMWPE can cause significant error while determining the weight loss. These are the main limitations of the gravimetric method [15, 16].

\section{Coordinate measuring machines}

CMMs have been widely used for dimensional inspection of complex shaped objects $[19,20]$. They can measure the dimensions with tactile probe or scanning probe $[21,22]$. This technique is one of the geometry based method that can be used both for evaluating wear volume and wear distribution over the worn surface [10, 11]. For defining the quantity of the wear, it requires unworn reference geometry of the prosthesis for comparing with worn geometry. In wear simulator studies the geometry of the specimen is defined before and after the wear test and then the results are compared for evaluating of the wear [17]. But it is difficult for retrieved implants because there is no pre-worn information about the implants geometries. In that case the unworn regions of the retrieved insert can be used for estimation of unworn geometry. It has been suggested that the minimum accuracy of a CMM for three-dimensional wear analyses should be $2 \mu \mathrm{m}[11,17,23,24]$. Bills et al. [17] proposed a CMM based technique for defining the volumetric wear of retrieved hip prosthesis. They took measurements from unworn region of the retrieved prosthesis and create 3D CAD model of idealized surface representing pre-wear surface of the prosthesis. Then they took points across the worn surface and produced surface wear maps. By comparing the two data sets they estimated the wear volume. 
Especially to determine the actual wear amount of UHMWPE it is important to define the creep deformation of the prosthesis. Besides wear measurements, CMM method provides volumetric and linear creep measurements [22, 25, 26]. Estok II et al. [25] measured the creep deformation of UHMWPE and cross-linked polyethylene by CMM method. They studied on two groups of acetabular components under physiological load conditions with out motion for defining the creep behavior. In another simulator study, Spinelli et al. [27] measured the wear of UHMWPE tibial inserts by CMM. They monitored the material behavior throughout the test to distinguish wear from plastic deformation and creep. They also used the gravimetric method as a reference for comparing the measurement results of wear. The authors concluded that however CMM technique overestimated the measurement values, two methods were in good agreement.

Rahmat et al. [22] used CMM laser scanner for digitizing complex shaped knee prosthesis. They proposed an algorithm to gain information from digitized data. Carmignato et al. [10] emphasize in their study that the uncertainty of wear volume, measured by CMM, has not been quantified yet. They used both gravimetric method and CMM technique for quantifying wear volume. They concluded that the CMM measurement results were in good agreement with gravimetric measurements. Besides this they evaluated the uncertainty of wear volume measurement from CMM data and gave suggestions to reduce the uncertainty. Although CMM technique gives advantages defining wear volume, wear scars distribution, and creep deformation, it is time consuming and needs uncertainty evolution for wear measurement.

\section{Micro computed tomography}

Micro-CT technique uses X-rays to generate three-dimensional geometries of the implants. By using calibrated X-ray detector the implants are scanned and then the data are processed by computer. This method provides reliable and accurate measurement of wear of UHMWPE but it is one of the expensive methods and has some difficulties to distinguish actual wear amount from creep. Like CMM technique the $\mu$-CT method requires unworn geometry of the prosthesis. Deviations from this initial geometry are used to define the $3 \mathrm{D}$ wear patterns. As mentioned before, for retrieval analysis it is difficult to find the unworn geometry of the implants $[13,18,28]$. To solve this problem, Teeter et al. [11] proposed reverse engineering method for defining an unworn reference geometry. They scanned 6 nonimplanted polyethylene tibial inserts by $\mu$-CT scanner. They obtained the surface meshes by reconstructing the scans and averaged the surfaces for creating reference geometry. They concluded that they successfully obtained idealized average $3 \mathrm{D}$ reference geometry representing of the true unworn geometry on the surfaces within $8.3 \pm 12 \mu \mathrm{m}$ where most wear occurs. In another study, Bowden et al. [13] studied on six retrieved UHMWPE acetabular components. These components were scanned by using high resolution $\mu$-CT scanner. They used rigid 3D image registration of the interior hemispherical portion of the acetabular cup with geometric primitives for extraction of volumetric wear. They also used acetabular components from simulator study as control specimen. These specimen wear was measured by gravimetric method and the results were compared. The authors concluded that uncertainty of the method was $0.6 \%$.

\section{Digital photogrammetry}

Digital photogrammetry is a method that measures $2 \mathrm{D}$ or $3 \mathrm{D}$ object's geometrical properties by using one of the three sources such as topographical maps, 2D photographic images and coordinate points of required object points. For 2D photographic images the worn prosthesis are digitally photographed by using a high resolution digital camera. An adjacent reference grid must be positioned at the same level of the prosthesis while taking digital photos. This is necessary for scaling the worn area. After taking images, each image is imported into software and scaled via its reference grid and the damaged area is measured. For creating 3D coordinates of the prosthesis the method uses two or more photographic images taken from different positions. Then these images are imported into software and evaluated [29-31].

This technique is independent from size and design of the implants. Actually it does not need unworn geometry of the retrieved prosthesis. It does not need complex and expensive machines. Shelf equipment is enough for application but the method needs improvement about repeatability and accuracy. For that reason Grochowsky et al. [29] studied on eighty retrieved UHMWPE tibial knee inserts for quantifying the accuracy and repeatability of wear area measurement by digital photogrammetry. They also evaluated the design dependence of their measurement methodology by studying on different insert designs. The authors concluded that the digital photogrammetry method could be used for measurement of wear. It was accurate, repeatable and design independent. In another study, Akbari et al. [31] studied on backside surface deformation in retrieved acetabular liners. They measured the surface changes by using $2 \mathrm{D}$ photogrammetry method. The authors concluded that, because of the minimal surface damage of the liners, the digital photogrammetry method was appropriate and more accurate for this study.

\section{Conclusion}

Measurement and evaluating of wear of retrieved implants provide useful information that can be used for developing design and material of implants. There are lots of current methods for defining wear of prosthesis such as radiographic, gravimetric, volumetric and optical techniques. Gravimetric method is the standardized method for quantifying wear volumes of total joint prosthesis. It is effective for determining experimental wear 
volume in simulated conditions but it is not suitable for assessing the clinically retrieved prosthesis because the method requires pre-wear data of the prosthesis. So the gravimetric method is usually used in simulator studies as a reference method. Although analyzing volumetric wear of retrieved implants by gravimetric, optical, and volumetric techniques can give accurate measurements values, they are time consuming processes and they give wear results including both creep and plastic deformation. They cannot separate the creep and wear amount of the UHMWPE. Actually each method has advantages and limitations. There are numerous studies about different wear assessment techniques. However accurate and repeatable results the techniques give about wear, they are not able to evaluate the results by giving wear amount, wear scars, creep, and plastic deformation. In conclusion, studies are going on for eliminating the limitations of the methods.

\section{References}

[1] W. Shibo, G. Shirong, L. Hongtao, X. Huang, J. Biomimet. Biomater. Tissue Eng. 7, 7 (2010).

[2] H. Bhatt, T. Goswami, Biomed. Mater. 3, 042001 (2008).

[3] J.M. Martell, E. Berkson, R. Berger, J. Jacobs, J. Bone Joint Surgery Am. 85A, 1111 (2003).

[4] E.P.J. Watters, P.L. Spedding, J. Grimshaw, J.M. Duffy, R.L. Spedding, Chem. Eng. J. 112, 137 (2005).

[5] S. Ge, S. Wang, N. Gitis, M. Vinogradov, J. Xiao, Wear 264, 571 (2008).

[6] D. Xiong, S. Ge, Wear 250, 242 (2001).

[7] L. Mattei, F. DiPuccio, B. Piccigallo, E. Ciulli, Tribol. Int. 44, 532 (2011)

[8] T. Mizoue, K. Yamamoto, T. Masaoka, A. Imakiire, M. Akagi, I.C. Clarke, J. Orthoped. Sci. 8, 491 (2003).

[9] T. Masaoka, I.C. Clarke, K. Yamamoto, J. Tamura, P.A. Williams, V.D. Good, H. Shoji, A. Imakiire, Wear 254, 391 (2003).

[10] S. Carmignato, M. Spinelli, S. Affatato, E. Savio, Wear 270, 584 (2011).

[11] M.G. Teeter, D.D.R. Naudie, J.S. Milner, D.W. Holdsworth, J. Arthroplasty 26, 497 (2011)

[12] International Standard ISO 14242-2:2000, Implants for surgery - wear of total hip joint prostheses. Part 2. Methods of measurement.
[13] A.E. Bowden, S.M. Kurtz, A.A. Edidin, J. Biomed. Mater. Res. Part B: Appl. Biomater. 75B, 205 (2005).

[14] K. Burckhardt, C. Gerber, J. Hodler, H. Notzli, G. Szekely, Med. Image Anal. 4, 375 (2000).

[15] P. Bills, L. Brown, X. Jiang, L. Blunt, J. Phys., Conf. Series 13, 316 (2005).

[16] L. Blunt, P. Bills, X. Jiang, C. Hardaker, G. Chakrabarty, Wear 266, 424 (2009).

[17] P. Bills, L. Blunt, X. Jiang, Wear 263, 1133 (2007).

[18] S.M. Kurtz, The UHMWPE Handbook: Ultra-High Molecular Weight Polyethylene in Total Joint Replacement, Elsevier Academic Press, London 2009, p. 511.

[19] M.N. Durakbasa, P.H. Osanna, Measurement 33, 157 (2003).

[20] M.N. Durakbasa, P.H. Osanna, A. Afjehı-Sadat, D. Samarawıckrama, A. Krsek, Measur. Sci. Rev. 5(2), 1 (2005).

[21] M.N. Durakbasa, Geometrical Product Specifications and Verification for the Analytical Description of Technical and Non-Technical Structures, Abteilung Austauschbau und Masstechnik, Wien 2003, p. 126.

[22] R.W.O.K. Rahmat, N.G. Seng Beng, K.A.P. Sangaralingam, Jurnal Teknologi 45(D), 97 (2006).

[23] O.K. Muratoglu, H.E. Rubash, C.R. Bragdon, B.R. Burroughs, A. Huang, W.H. Harris, J. Arthroplasty 22, 435 (2007).

[24] L.A. Blunt, P.J. Bills, X.Q. Jiang, G. Chakrabarty, Proc. Inst. Mech. Eng. H. 222, 309 (2008).

[25] D.M. Estok, II, C.R. Bragdon, G.R. Plank, A. Huang, O.K. Muratoglu, W.H. Harris, J. Arthroplasty 20, 239 (2005).

[26] O.K. Muratoglu, C.R. Bragdon, B.S, Murali Jasty, D.O. O'Connor, R.S. Von Knoch, W.H. Harris, J. Arthroplasty 19, 887 (2004).

[27] M. Spinelli, S. Carmignato, S. Affatato, M. Viceconti, Wear 267, 753 (2009).

[28] M.G. Teeter, D.D.R. Naudie, K.D. Charron, D.W. Holdsworth, J. Arthroplasty 25, 330 (2010).

[29] J.C. Grochowsky, L.W. Alaways, R. Siskey, E. Most, S.M. Kurtz, J. Biomed. Mater. Res. Part B: Appl. Biomater. 79, 263 (2006).

[30] R. Bogue, Sensor Rev. 30, 102 (2010).

[31] A. Akbari, M.E. Roy, L.A. Whiteside, B.J. Katerberg, D.J. Schnettgoecke, J. Arthroplasty, in press. 